\title{
Hierarchical Co,Fe-MOF-74/Co/Carbon Cloth Hybrid Electrode: Simple Construction and Enhanced Catalytic Performance in Full Water Splitting
}

\author{
Qingqing Zha, Mingxia Li, Zihao Liu and Yonghong Ni*
}

College of Chemistry and Materials Science, Key Laboratory of Functional Molecular Solids, Ministry of Education, Anhui Laboratory of Molecule-Based Materials, Anhui Key Laboratory of Functional Molecular Solids, Anhui Normal University, 189 Jiuhua Southern Road, Wuhu, 241002, PR China. Fax: +86-553-3869303. E-mail: niyh@mail.ahnu.edu.cn

Page number: 6

Figure number: 12

Table number: 0 

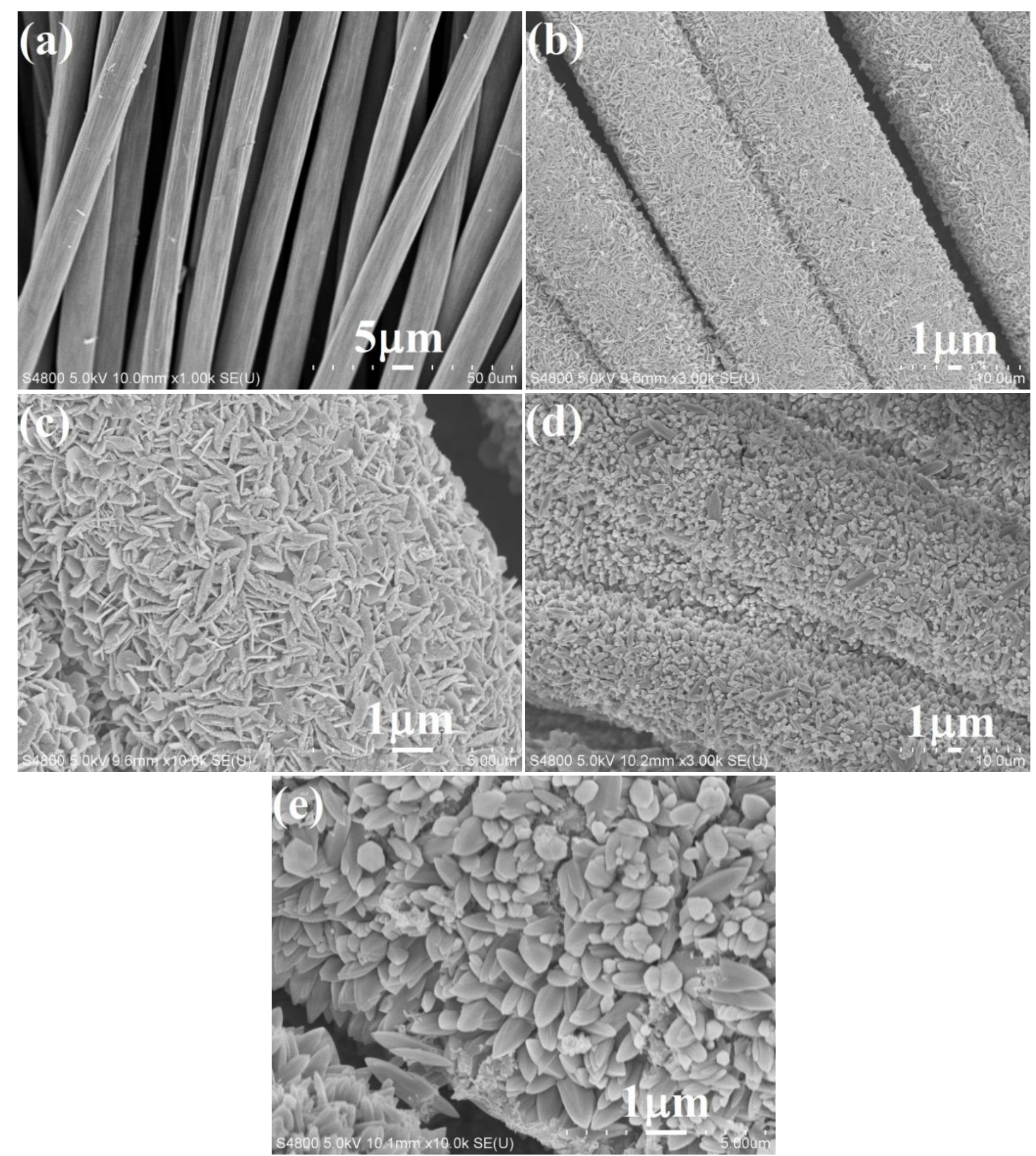

Figure S1. FESEM images of the bare CC (a), the Co/CC (b,c) and the Co-MOF-74/Co/CC (d,e).

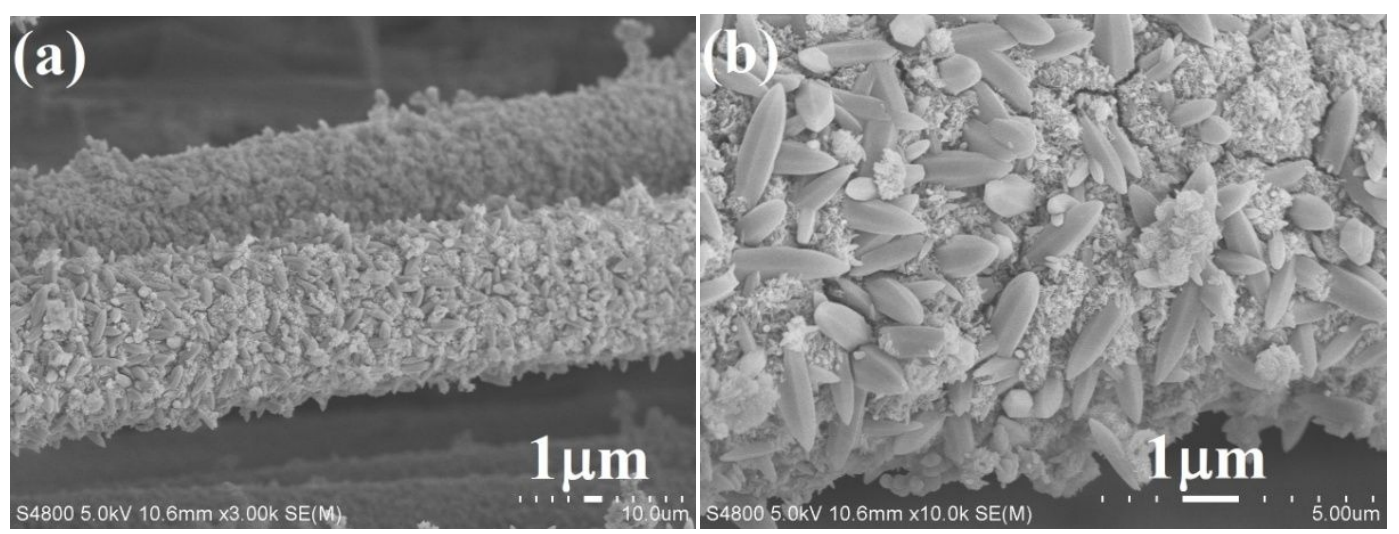



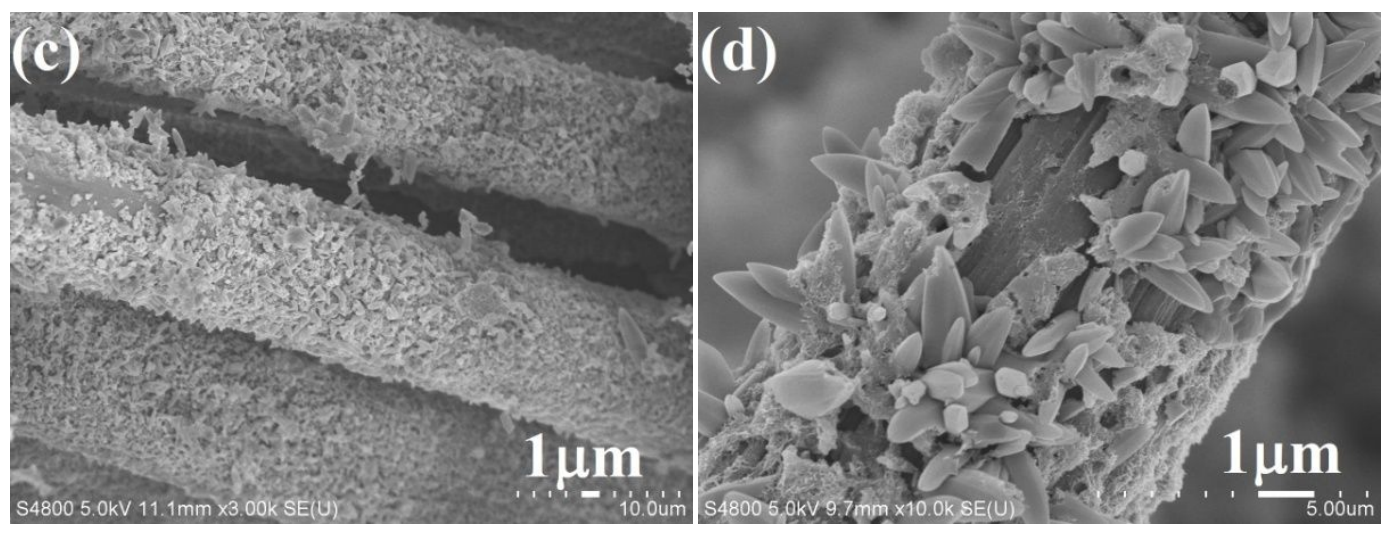

Figure S2. FESEM images of Co,Fe-MOF-74/Co/CC prepared from the systems with different initial amounts of $\mathrm{FeCl}_{3} \cdot 6 \mathrm{H}_{2} \mathrm{O}:(\mathrm{a}, \mathrm{b}) 1 \mathrm{mg}$ and (c, d) $3 \mathrm{mg}$.

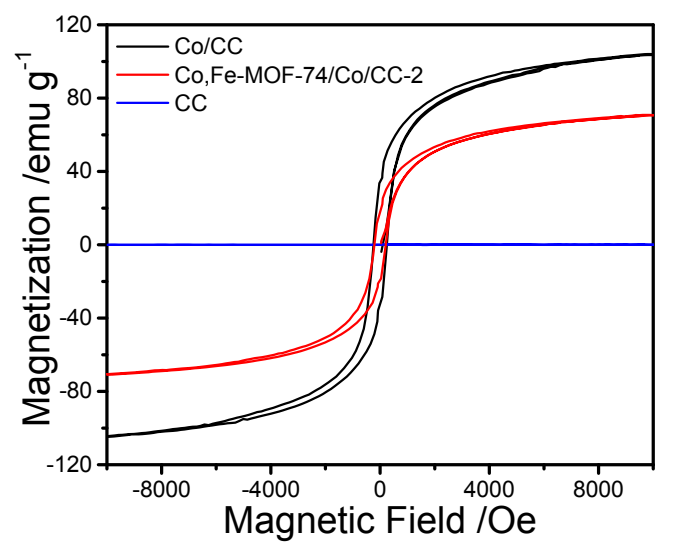

Figure S3. The room temperature hysteresis loops of $\mathrm{Co} / \mathrm{CC}, \mathrm{Co}, \mathrm{Fe}-\mathrm{MOF}-74 / \mathrm{Co} / \mathrm{CC}-2$ and CC.
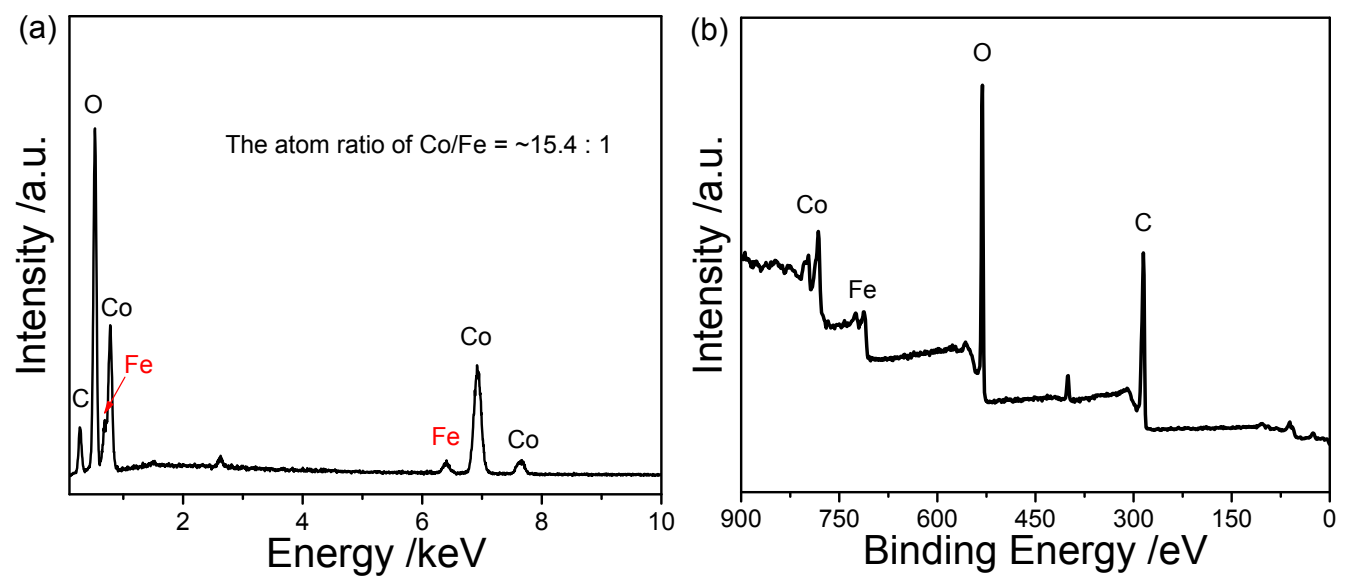

Figure S4. (a) EDS and (b) XPS survey spectrum analyses of as-obtained Co,Fe-MOF-74/Co/CC. 


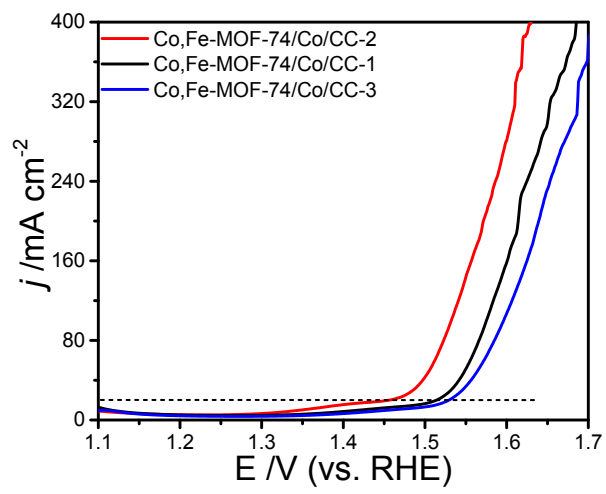

Figure S5. Polarization curves of the final products prepared under the same conditions with various amounts of $\mathrm{FeCl}_{3} \cdot 6 \mathrm{H}_{2} \mathrm{O}(1,2$ and $3 \mathrm{mg})$.

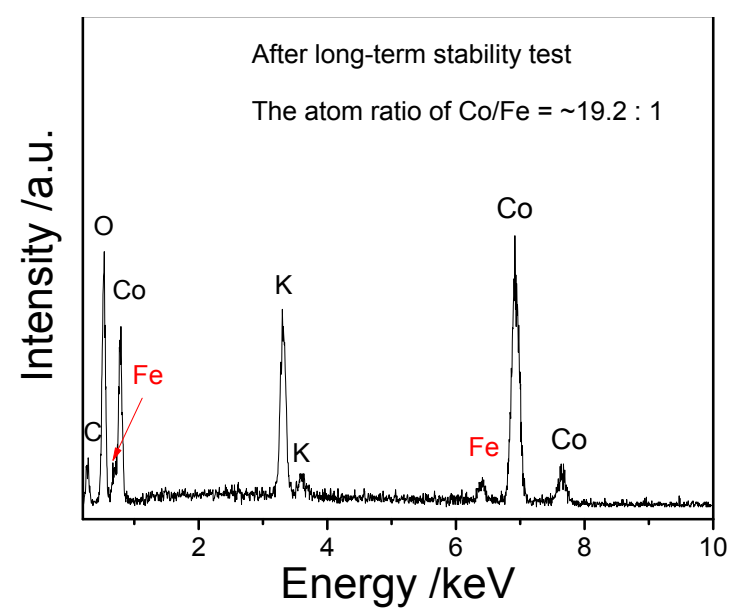

Figure S6. EDS analysis of $\mathrm{Co}, \mathrm{Fe}-\mathrm{MOF}-74 / \mathrm{Co} / \mathrm{CC}-2$ after the $70 \mathrm{~h}$ durability test.
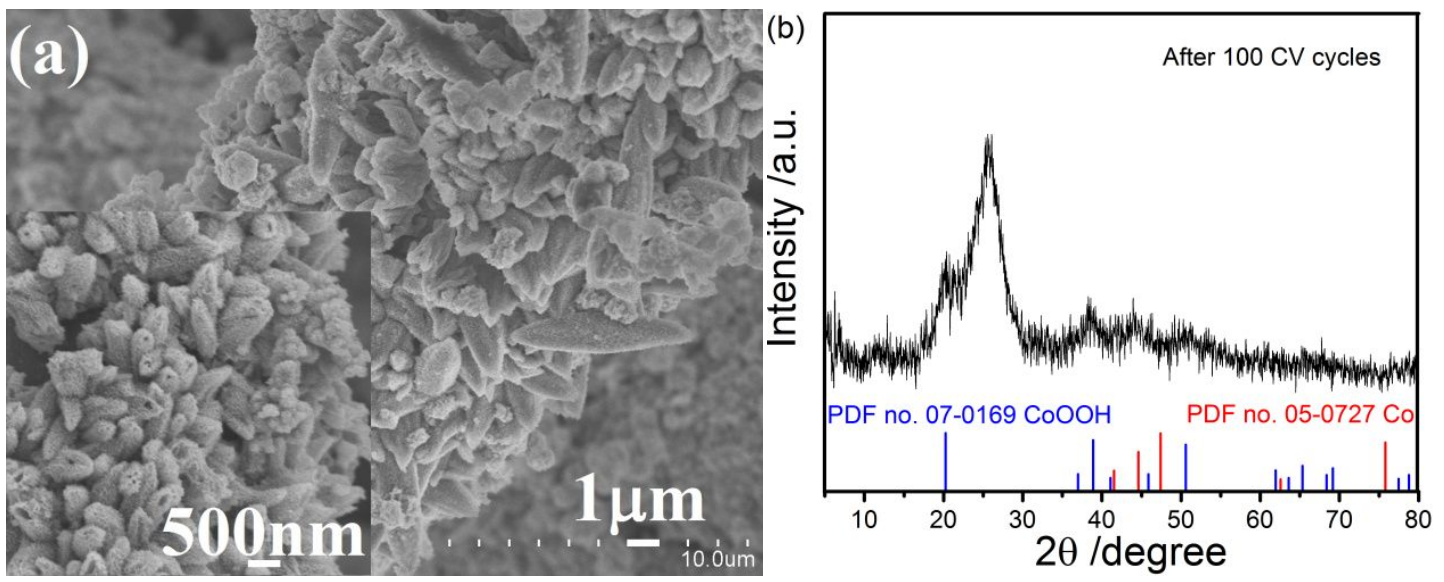

Figure S7. (a) FESEM image (b) and XRD pattern of the Co,Fe-MOF-74/Co/CC-2 electrode after $100 \mathrm{CV}$ cycles for OER in $1 \mathrm{M} \mathrm{KOH}$ solution. 

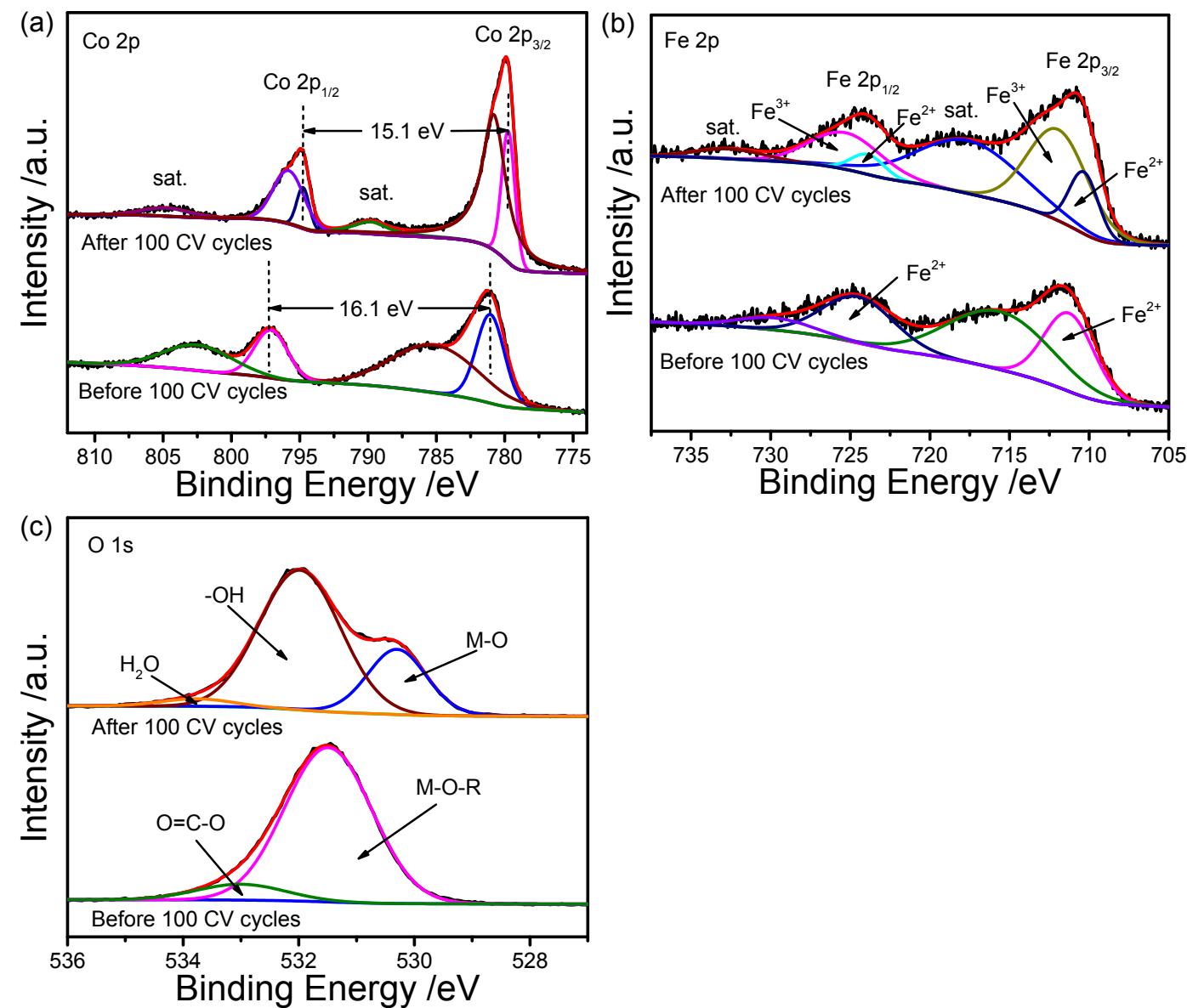

Figure S8. XPS analyses of Co 2p (a), Fe 2p (b) and O 1s (c) before and after $100 \mathrm{CV}$ cycles.
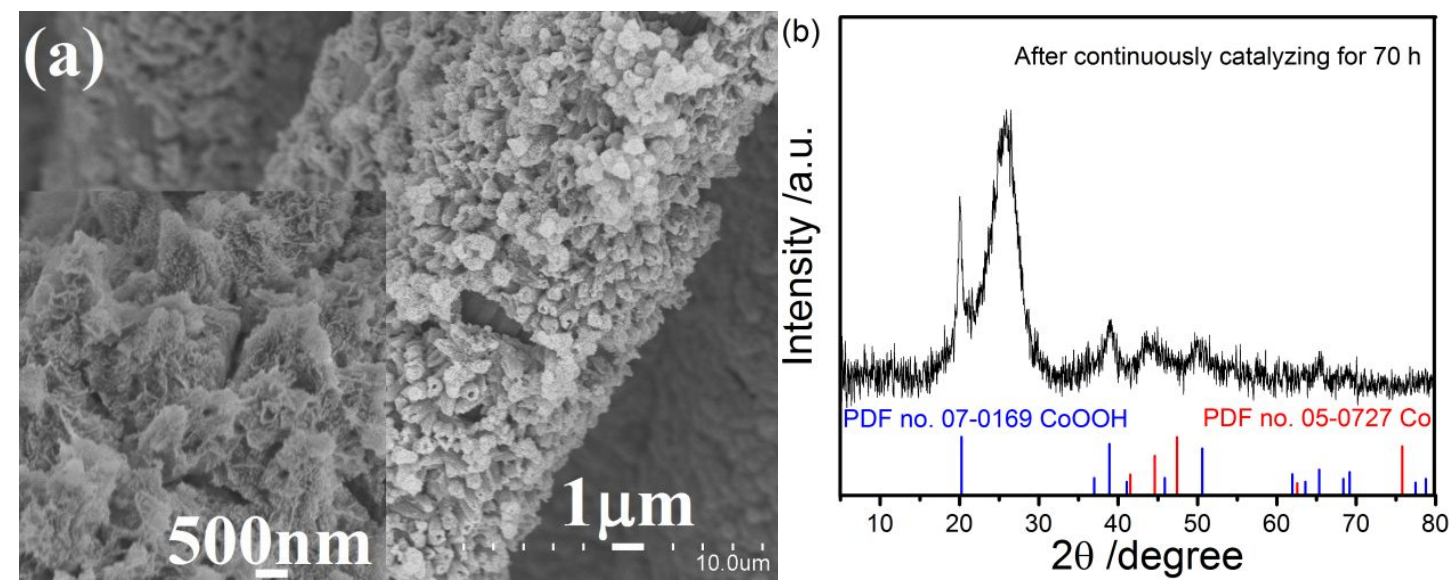

Figure S9. (a) FESEM image and (b) XRD pattern of the Co,Fe-MOF-74/Co/CC-2 electrode after continuously catalyzing for $70 \mathrm{~h}$ in $1 \mathrm{M} \mathrm{KOH}$ solution at the current density of $20 \mathrm{~mA} \mathrm{~cm} \mathrm{~cm}^{-2}$. 

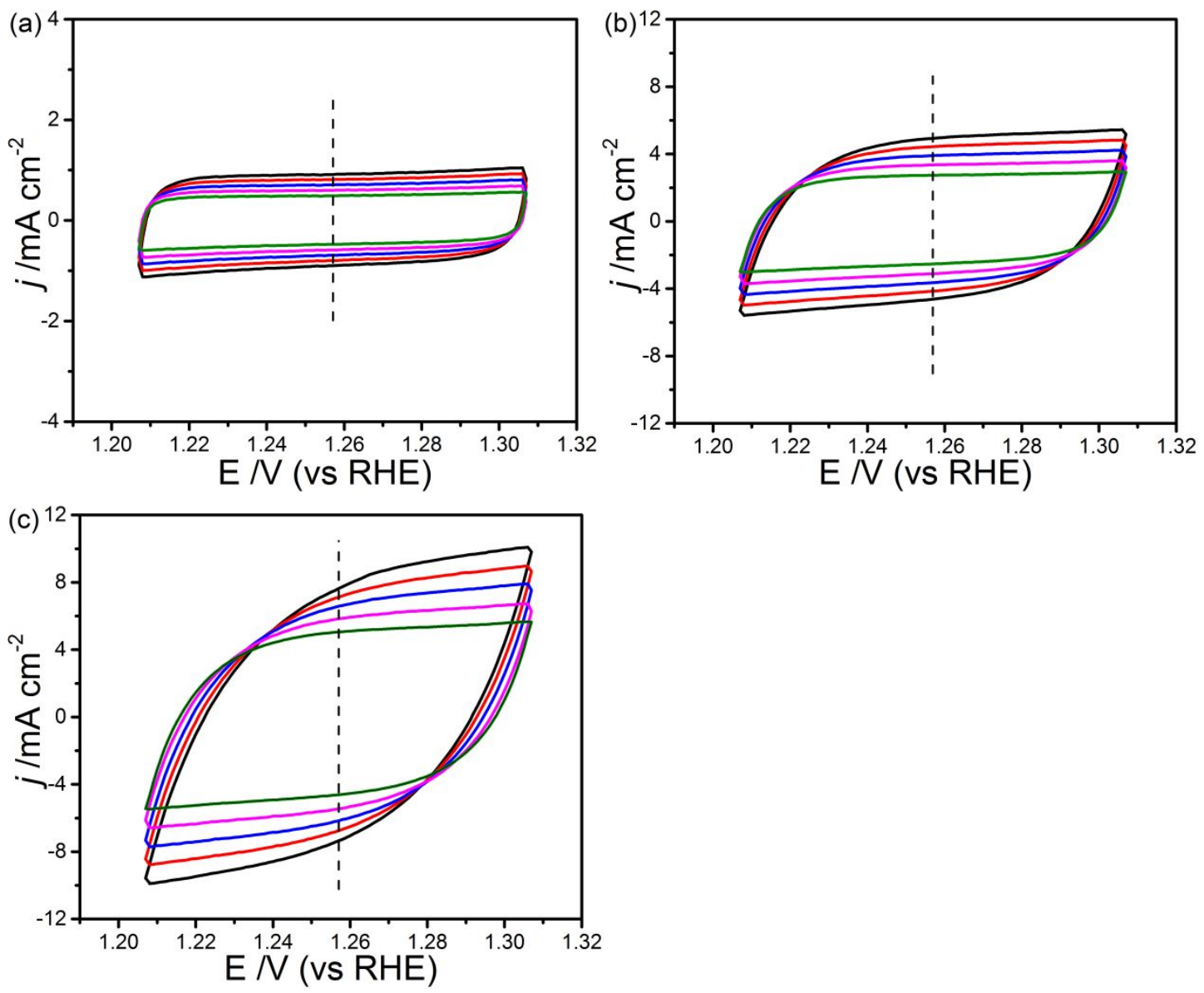

Figure S10. CV curves of various electrodes in the potential window from 1.207 to $1.307 \mathrm{~V}$ (vs.RHE) at 20, 25, 30, 35 and $40 \mathrm{mV} \mathrm{s}^{-1}$ : (a) $\mathrm{Co} / \mathrm{CC}$, (b) Co-MOF-74/Co/CC and (c) Co,Fe-MOF-74/Co/CC-2.
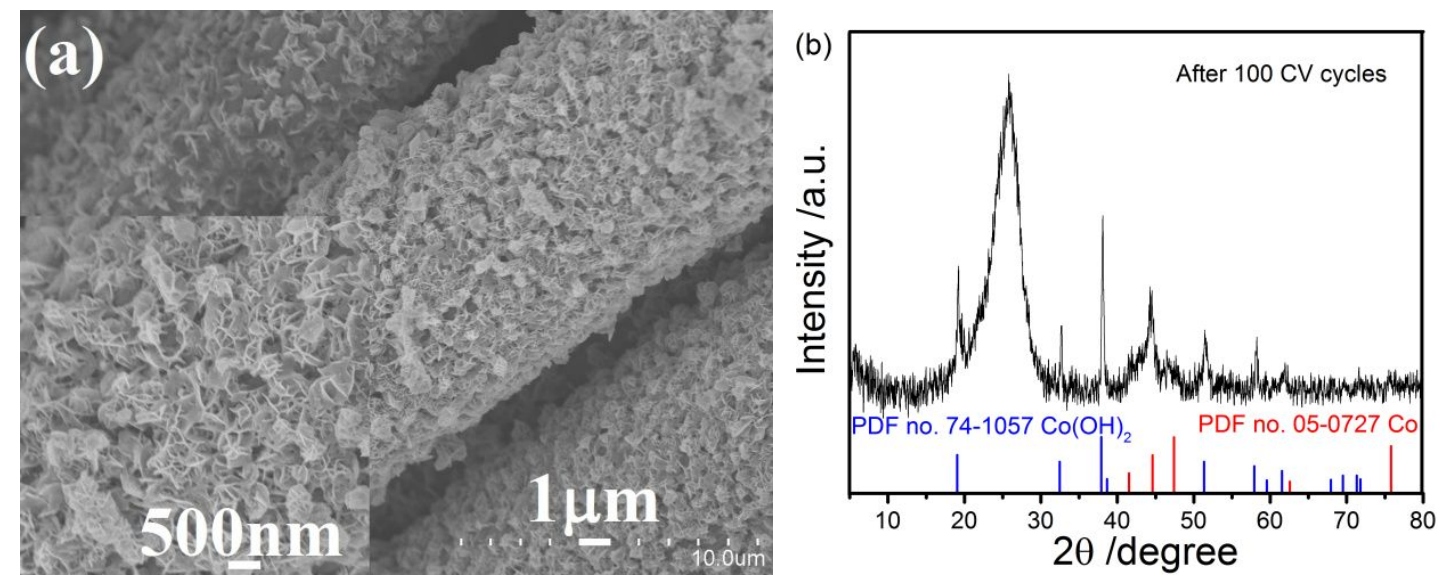

Figure S11. FESEM image (a) and XRD pattern (b) of the Co,Fe-MOF-74/Co/CC-2 electrode after $100 \mathrm{CV}$ cycles for HER in $1 \mathrm{M} \mathrm{KOH}$ solution. 


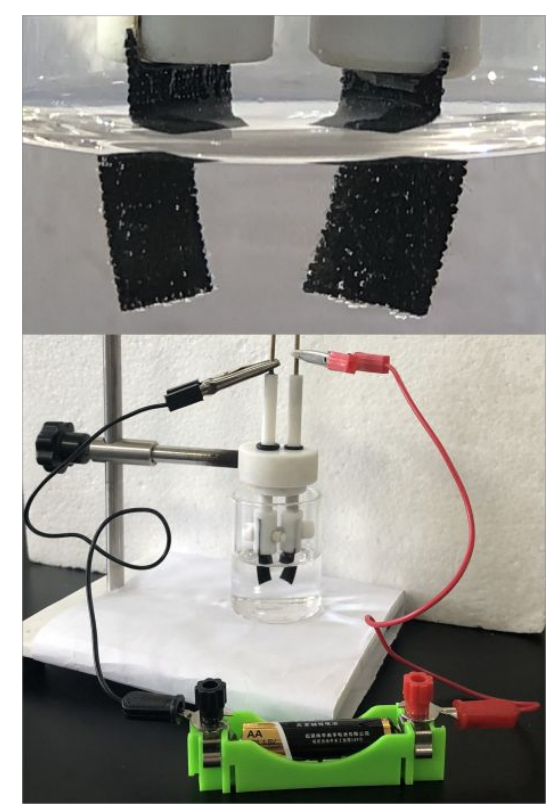

Figure S12. The photographs of water electrolysis driven by a commercial AA battery of $1.5 \mathrm{~V}$ with the $\mathrm{Co}, \mathrm{Fe}-\mathrm{MOF}-74 / \mathrm{Co} / \mathrm{CC}-2$ electrode as both anode and cathode. 